\title{
ESTUDIO MACRO-MICROSCOPICO Y ENZIMATICO CUALITATIVO DE CEPAS MICELIALES DE BASIDIOCARPOS DE AGARICALES II.
}

\author{
(A qualitative macro-microscopic and enzymatic study of mycelial strains from \\ basidiocarps of Agaricales II.)
}

\author{
Garnica, S. \& Valenzuela, E. \\ Institulo de Microbiologia. Facultad de Ciencias, \\ Universidad Austral de Chile. Casilla 167, Valdivia.
}

Palabras clave: Cepas miceliales. cullivo puro. morfologia. cnzimas. Agaricales, Chile. Key words: Mycelial strains, purc culture, morphology, enzymcs, Agaricales, Chile.

\section{RESUMEN}

Se estudiaron en cullivo puro las cepas miceliales de 8 especies de Agaricales de la madera que fructifican en el centro-sur de Chile. Las cepas miceliales: UACHALAp480 de Agrocybe praecox. LIACHM Lal-t7t de Amhracophyllam discolor, $U_{A}-\mathrm{C} C \mathrm{C} / \mathrm{C} b-++2$ de Collybia butyracea, L.ACHMCg-193 de Collybia grinhergsii, UACHA IDa-t0.5 de Descolea antarctica, U.ACHAMP/-26.5 de Panellus longinquas, LACH/PCr-280 de Pleuroflammula croceosanguincay UACHM $/ P^{\prime n-2+0 ~ d e ~ P l u t e a s ~}$ nanus, fueron obsenidas descle el pseudotejiclo de los basidiocarpos correspomdientes. Se discuten algunos de los caracteres macro-microscópicos de los micelios desaroollados sobre losmedios de cullino PD. I, AEN I, CzA y la deleccion cualitativa de sus enzimas.

\section{INTRODUCCION}

Sc aportan mucros antecedentes sobre las calactcrísticas macro-microscópicas y cm\%imáticas cualital ivas de ccpas miceliales aisladas y desarrollaclas cn cullivo puro a partir de basidiocarpos de 8 especies de Agaricales que fruclifican sobre diversos sustratos lcĩosos del centrosur de Chilc. Otras contribuciones que hemos realizado sobrc la caracicriación morfológica y bioquímica cualitativa de micelios de Agaricales que viven sobre sustratos lignocclulósicos corresponden a Garnica (1995). Gárnica et al. (1997) y' Valcuzucla et al. (1997).

\section{MATERIALES Y METODOS}

Las 8 cepas miceliales caraclerizaclas en el presente estudio fucron obıcnidas desde el pscudolcjido de basidio-

\section{SUMMARY}

Mycelial strains of eigth species of wood-inhabiting Agaricales that fructify in the center-south of Chile were studied in pure culture. The following mycelial strains: UACHM.A p-t80 from Agrocybe praecox, U.4CHNLAd-474, from Anthracophyllum discolor, UACIIA/C.b-+12, from Collybia butyracea, UACHM $\mathrm{Ag}-193$ from Collybia grinbergsii, UACHM $D$ Da- 405 from Descolea antarctica, UACIA IIP-26.5 from Panellus longinquus, UACHMPCr280 from Pleuroflammula croceosanguinea and U.1CHN/Pn-2 40 from Pluteus nanus were obtained from the pseudotissue of the corresponding basidiocarps. Some of the macro-microscopic characteristic of mucelial developed in PDA , MEA CzA culture media as well as the qualitalive detection of their enzymes are discussed.

carpos de Agaricales de madera, que fructifican en el cenrro-sur de Cliilc. Los basidiocarpos fucron desinfectados superficialnente con clanol al $96 \%$ y desde la zona entre pilco y cslipite, sc c.xtrajcron asćplicamente 10 trozos de pscudolcjido de $0.3 \mathrm{~cm}^{2}$ que se sembraron en placas Petri que conlcnían agar cxtraclo de malla al $2 \%$, adicionado de antibiólico y se incubaron a $23^{\circ} \mathrm{C}$ por 7 días (Molina \& Pailmer 1982).

Para delcrminar las características de cultivo, se exirajeron trozos de agary micel io de $0.6 \mathrm{~cm}^{2}$ de diámetro de las discintas ccpas. los cuales fueron inoculados independientcmcnte, cn cl centro de placas Petri que contenían los medios de cullivo PDA, AEM y CzA al 2\% y se incubaron a $23^{\circ} \mathrm{C}$. Para la caraclcrización mor rológica y morfomćtrica, los cultivos desarrollidos en los medios mencionados, se 
examinaron a los 7 y $1+$ días. confcccionándosc preparaciones al fresco del micclio de diferentes zonas de la colonia, usándose agua como líquido de montajc. La forma y textura de las colonias se establecicron de acuerdo a lo scñalado por Nobles (1948. 1958. 1965) y Stalpers (1978). La detección cualitativa de las enzimas se ejecutó a los 14 días de cultivo: para las cuzimas citocromo oxidasa, esterasa, fosfatasa, lacasa. peroxidasa y lirosinasa se siguió el procedimiento descrito por Taylor (1974) y Stalpers (1978). En la detccción de las cnzimas amilolílicas, celulolíticas y pectinóliticas se usó una lécnica modificada de Pochon \& Tardicux (1965). La enzima oxidasi cxiracclular se delccló de acucrdo a Noblcs (1958). Micntras que las enzimas ureasa y protcolíticas se detcrminaron scgún lo descrito por Mac Faddin (1976) y las cnzimas DNAsa y lipasa como indican Hankin \& Anagnoslakis (1975). Se utilizaron como cepas controles Trumetes versicolor Schlcchı (Tv) y Fusurium oxysporum Schlccht.:Fr., proporcionadas por cl Centraalburcau roor Sclummel Cultures, Baarn, Holanda.

Las cepas miceliales son indicalas en el texto con Ja sigla UACHM seguidas de las iniciales de la cspecie correspondiente y el número de colccla. Tanto los basidiocarpos, asi como las cepas miceliales se encuentran depositados en el Herbarió y cepario del Instiluto de Microbiologia de la Universidad Austral de Chile. ValdiviaChile.

\section{RESULTADOS}

1.- Caracterización macro-microscópica de las cepas miceliales de Agaricales en cultivo puro.

\section{A) Cepa UACHMAp)-480}

Obicnida de basidiocarpos de Agrocybe praecox (Pcrs.: Fr.) Fayod., recolcclados desde locón de Norhofugus dombeyi, «coiguc». Popoćn, Osorno 27-V-95.

\section{Medio PDA}

Macroscopía: Colonia de 56 mm de diám. circular y zonada; subfclposa-felposa a vcllosa, blanquecina. Margen apresado (igual en CzA). Reverso amarillo-rerdoso. Olor a «humedad» (igual cn AEM).

Microscopía: Hifas de (1.4 ) 2.8-5.6 (-7) pun de diám., (igual en AEM). fibuladas, entrclazadas o bien terminadas en estrucluras similares a glococislidios. Hifas de $1.4-2.8(+.2)$ $\mu \mathrm{m}$ de diám., de crecimiento limitado. frecuentes en cl centro de la colonia. color marrón (Fig. 1).

\section{Merlio AEM}

Macroscopía: Colonia de 79) mm de diám., circulary zonada; textura sub/clposa-l'cllosa (algo farinácea), blanquecina a cremosa. Margen apresido. Reverso amarillento. Olor «ha- rinoso»

Microscopía: Hifas aćrcas algunas entrelazadas café-amarillentas. Hifas apresadas de mayor diámetro que las hifas aćrcas, liguradas.

\section{Medlio CzA}

Macroscopía: Colonia de 81 mm de diám., circular; textura flocosa-plumosa, grisácea. Inodonas.

Microscopía: Hifas de $1.4-4.2 \mu \mathrm{m}$ de diám., fibuladas. algunas anastomosadas. Artroconidios poco frecuentes de $5.6-1+\times 1.4-2.8 \mu$ un.

\section{B) Cepa UACHMA(l-474}

Oblcnida de basidiocarpos de Anthracophy/lum discolor (Mont.) Sing., recolectados desde restos de ramas en descomposición de Myrceugenia parvifolia "palaguilla». Arboretum de la Universidad Austral de Chilc. Vildivia 30-III-95.

\section{Medio PDA}

Macroscopía: Colonia de 16-30 mm de diúm., circular: testura subfclposa, café-claro a amarillenta. Margen apresaldo. Rcicrso cafć a crómco-amarillcnto. Olor frutal irritante. Microscopía: Hifas de 1.4-2.8 (-4,2) pum de diám. Fíbulas prescntes. a veces abiertas. Hifas de (2.1) 2.8-4.2 $4 \mathrm{~m}$ de diám. de parcdes ramificadas algo verrucosas. Fibulas prescnles, a veces abicrlas. Hifas de $2.8 \mathrm{~mm}$ de diám., sin septos y con estructuras similares a fibulas, frecuentes a lo largo de la hifa. Hifas incrusladas con matcrial similar a costrass o placas, de color café a café-rojizo (igual en AEM) (Fig. 2). Hifas entrclazadas de color cafć-amarillento (igual en AEM). Cristales, rectangulares y otras formas, refringentcs, cáfć a cafć-rojizo (igual en AEM).

\section{Medio AEM}

Macroscopía; Colonia de 14 mm de diám., (olros caracteres igual que en PDA). Reverso crémco-amarillento a amarillopálido. Inodoras.

Microscopía: Hifas con dilatacionesde 7-9.8 8 5.6-7 mm, hialinas. Clamidosporas intcrealares y tcrminales de 7-15.4 x.5.6-7 $\mathrm{mm}$ (Fig. 3).

\section{Meclio CzA}

Macroscopía: Colonia de $8 \mathrm{~mm}$ de diám., circular; textura escasamentc farinácea a pelicular, blanquecina. Margen apresado. Inodora.

Microscopía: Hifas de 1.4-2.8 (4.2) mmm de diám., hialinas, vacuolizadas y ramificadas. Fíbulas presentes. Hifas entrclazadas o bien coraloides. Cristales hexaćdricos.

\section{C) Cepa UACHMCb- +42}

Obicnida de basidiocarpos de Collybia butyracea (Bull.: Fr.) Kummer, recoleclados desde madera en descom- 
posición de Nothofagus oblitua, «roblen. Popoćn. Osomo II-XII-)t.

\section{Medio PDA}

Macroscopía: Colonia de 9 ) mun de cliann. (igual cn AEM) circulary \%onlada: Icvtura lanosia a algodonosia. blinncuccina. Margen Icranulaclo. Rererso blanquecino a antarillentopílido Olor débilmente dul\%aino.

Mlicroscoppía: Hi Fas aércils de $2.8-11.2 \mu$ de diain.. algo ramilicadlas. de paredes gruesals refringenics. Fibulas simples y múlliples (iguil cn AEM) (Fig. 4). HiFis de I. t-2.8

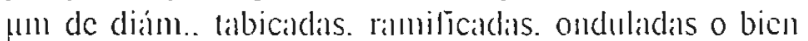

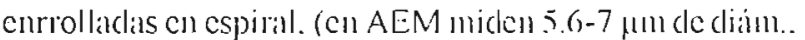
de paredes grucsis hasta 2.8 fum). ribulis anuscntes.

\section{Medio AEM}

Macroscopia: Colonia irregular yonada. blanci-imarillcnla con bandals concénuricals de distimtos gratos de intensidad. hacia el margen blanquecina a ligeramente grisiceal Icxlura (igual que en PDA). laicia cl margen con zonas aislaclas subfelposas ( Farimiceas). Margen Iciantado a apresado. Reverso cremoso con árcas irregulares de color cilfeamarillento. Inodoro.

Microscopía: - Hi las aćrcas do t.2-7 fun diám.

\section{Merlio C 2 A}

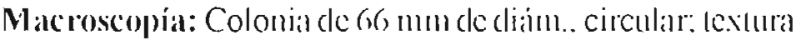
plumosit-flocosia a sub[elposit-peliculiar. blanquecinal a grisiccil. Reverso blanculecino. Olor dulzilino.

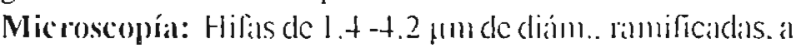
reces de palredes gruesis. rerringenles. escasimuculc labicadas y ramificadas. conrolladas con cspiral. con ramificaciones cortas o bien entrelizadias. Fíbulas ausentes. Cristales jumo a la hifa o aislados.

\section{D) Cepa UACHNI(g-t)3}

Obıcnicla de basicliocarpos cle Collybia grimbergsii Valcmzucla \& Moreno. recolectados desede rambis en descomposición de Nothofuzus obliqua, uroble-lualle». Arboremun de la Universidad Aust ral de Chile. Valdivial 1.3IV-95.

\section{Meclio PDA}

Macroscopía: Colonia de 15-26 mun de diám. irregulatry zonadai: Icxilura costrosil a scclosit-subfelposil. calfé-claro. Margen apresildo. Reverso cremoso. Inodora.

Nicroscopía: - Hifas ele ( 1.4$) 2.8-4.2 \mu u n$ de diain.. con corlas raminificaciones laterales. fibuliadlas (cn AEM miden has-

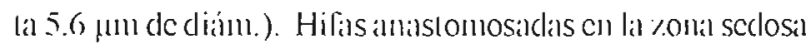
de $2.8-4.2 \mu$ de diám.. de paredes refringentes. hialinas o de color café amarillenlo (en AEM miden $1 .+-5.6$. 1.11 de diám). Hifas con dilataciones intcrealares y terminales de (5.6) $8.4-5.6 \times 12.6(1+4) \mu m$. Clamiclosporals incrcalares ! tcrminales de (5.6) 9.8- $1+x(+.2) 7-12.6 \mu m$. Crislales presentes.

\section{Merlio AEM}

Macroscopia: Colonia de $53 \mathrm{~mm}$ de diám.. irregular: Icvtura farinícci-subfclposia a sedosit, blanquecinal a calfé-crémco. Margen ilpresialo. Reverso cafć-claro a amarillo. Inodoro. Microscopía: - Hifas esqueléticas de 1.+ (2.1) pun de diám.. escisamente ramificadals. Clamidosporas intercalares y tenninales de $7-15.1 \times 5.6-1+\mu m$.

Medio Cr.A. No hubo crecinticnto.

\section{E) Cepa UACHMDa-4115.}

Oblenida de basidiocarpos de Descolea antarctica Sing.. recoleclados desde restos de cortèa depositada cu cl suclo de Nothofugus dombè i «coigue». Jardin Botánico de la Univcrsidad Auss ral de Chile. Valddivia 25-VI-94.

\section{Me(dic) PDA}

Mlacroscopía: Colonia de 9 ) mun de diám.. (igual cn AEM y C\%A) circular: 1extuma fanosit-subfelposia a al godonosa, blanquecina a cremosa. Margen leranlado (igual cn AEM). Rererso blingluccino. Olor dulzaino.

Microscopía: Hifiss de 1.4-9.8 $\mu m$ de diánl.. varicosas 0 cntrelazadass. hial linas a cafć-anlarillentas. Fibulas prescnIcs. Hifis libicadas. algumas con malcrial refringente en su inlerior o bien con pequenialas placas cpiparictalcs, sin fibulas. - Hifias con dilalaciones intercialares de | $1.2-18.2 x$

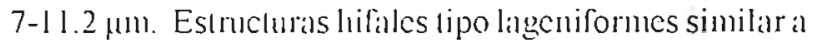
glococisciclios de 23.8-7() $\times 1$ 1 $1.2-1+\mu$ m. predominantes $\mathrm{cn} \mathrm{cl}$ cenll ro de la colonia (Fig. 5). Artroconidios de $+.2-21 \times 2.8-$ 8. $+\mu$ m (igual en AEM y C CA) (Fig. 6).

\section{Merlio AEM}

Macroscopia: Colonia irregular y zonada: Icxluna felposalanosal a vellosi. cremosa a blanquecina. Reverso blanquecino a almarillonlo. Inodoro (igual $\mathrm{cn} \mathrm{C} z \mathrm{~A}$ ).

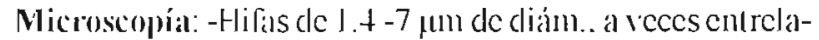
zaldas. incrustadis con milterial refringente y ranificadas. fibuladlas. Hilas frecuentemente tabicadlas. sin fibulas. Crisliles presentes (ignial en C\%A).

\section{Merdio Cr.A}

Macroscopia: Colonia plumosa-flocosa. lacia el margen pelicular. hialina a grisícca. Margen apresado. Reverso grisícco.

Nicroscopía: Hifas de $1 .+-7 \mu m$ de diám. a veces cntrelaraclas. onclulaclas. corrugadas y libuladas.

\section{F) CepaI UACHNIPI-26.5}

Oblenidal de basidiocarpos de Pane/lus longinquus (Berk.) Sing.. recolectiados descle tronco scco y' en des- 
composición de Salix babjlonica "Saucc». Jardin Bolánico de la Universidad Ausi ral de Chilc. Valderia 9-VI-95.

\section{Medio PDA}

Macroscopóa: Colonia de + mun de diam., irregular: Icrluna vellosa. blanquccina-crémca. Margen Icrantado (igual cn AEM). Reverso crémco. Inodoro (igual cn AEM y C $\% A$ ). Microscopía: Hifas de $1 .+-4.2 \mu$ diám. a veces anaslomosadas, ramificadas. hialinas a café. fibuladis. Crislalcs presenles.

\section{Merlio AEM}

Macroscopía: Colonia circular de 6 mm de diám. vellosa. grisácca. Reverso negruzco a grisácco.

Microseopría: - Hifás de 1 . anastomosadas.

\section{Me(lio) C.A}

Macroscopía: Colonia de 8 mm de diám.. inegular: Icrlura pelicular. blanquecina. Margen apreside.

Microscopía: Hifas de $1.4-2.8$ mm de diám.. algumas incrusladas con matcrial cristalino.

\section{G) Cepa UACH MIPer-280}

Oblenida de basidiocarpos de Pleuroflammula croceosangminea (Mont.) Sing. recolcclaclos desde ramas cn descomposición de Nothofugus dombeyi "coiguc». Rcbcllín. Valdivia 3()-V-94.

\section{Medio PDA}

Macroscopía: Colonia de 9() mun de cliám. circular Y \%onlada (igual en AEM): lextura felposa-farinaicea a algodonosa llegando a ser subfelposa-nocosi. blancucciua a cremosa. Margen apresado y rercrso blanquecino (igual cn AEM). Olor a Cyllaria (igmal cn AEM).

Microscopía: Hifas gencratias de $1 .+-+.2 \mu m$ de diánı. a veces con corlas ramificacioncs. Tibulas cscasas. Hifas esquelélicas de $1 .+-\downarrow .2 \mu m$ de cliám. cscilsimente ramificadas. a veces en el micelio aćreo presculan dilalaciones intercalares y icrminales de $+2-8 .+x+.2-7 \mu m$, hialinas (igual cn AEM). Artroconidios de 2.8-9.8. $1.4-2.8 \mu \mathrm{m}$.

\section{Medio AEM}

Macroscopía: Texlura de la colonia hacia el centro algodonosa. hacia cl margen felposa-farinácea cremosa a blanquccina.

Microscopía: Hifals gencrativas de $1.4-4.2 \mu u n$ de diám. a a reces corrugadas. ondulaclas y con corlas ramificaciones (cn C\%A miden hasla $5.6 \mu$ m). Fibulas cscasas. Hifas esquelélicas de $1 .+-2.8 \mu m$ de diam.. con dilataciones intercalares y terminales de 2.8-9.8 $\times 2.8-9.8 \mu 11$. (Fig. 7). Cristales prescnles (igual cn $\mathrm{C} \% \mathrm{~A}$ ).

\section{Medio CzA}

Macroscopía: Colonia de (66-76 mun de diám.. circular; texIura pelicular a flocosa-plumosa. blanquccina. Margen con hifas apresadas y distantes. Olor dulyaino.

Microscopía: - Hifas esquelćlicas de $1.4-2.8$ fum de diám.

\section{H) Cepa UACHMPn-240.}

Oblenida de basidiocarpos de Pluteus numus (Pers.: Fr.) Kummer ss Orlón. recolectados desde corleza de tronco de Nothofagus obliqua, "huallc" vivo. Rebcllin. Valdivia, 3()-V-94.

\section{Me(lio PDA}

Macroscopía: Colonia de 10 mun cle diám.. irregular (igual

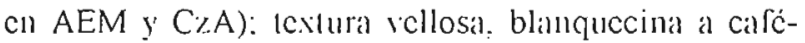
crémosa. Margen apresado (igual cn AEM y C 7.A). Alrededor de la colonia cl medio de cultivo sc toma cafć-ıcnuc. Por el reicrso cl centro café y hacia cl margen blanquecino-amarillcnto. lucgo manrón. Inodoro (igual cn AEM). Al mes de cultivo aparecen escasas y pequeñas rizomorfas. inmersas en cl agar de color câf́c.

Microscopía: Hifas de $1 .+-\$ .2 \mu m$ de diám. a veces con dilalaciones intercalares y terminales de 7-16.8 x + $+2-11.2$ 111 , sin fíbulas (igual cn AEM). Al mes de cullivo se obscrvaron células culiculares, ovoides, globosas. hialina a calć. Hifas incrustadas con malcrial cristalino cpipariclal y con diminulas proyeccioncs similares a «cllos». hialimas a carć.

\section{Medio AEM}

Macroscopía: Colonia de icxilura costrosal a vellosa. blanquecina. Rerciso calé-blanquecino.

Mlicroscopía: Como cu PDA.

\section{Medio C.A}

Macroscopia: Colonia de lcxilura costrosa a pelicular. El inóculo se lorna de color grisícco a calé, micclio pelicular, grisícco. Rererso negruzco.

Microscopría: Hifas varicosas a moníliodes cle $2.8-4.2 \mu m$ de diám. labicadas. con dilataciones de $5.6-1+x .5 .6-12.6$ fum (Fig. 8). Fíbulas ausentes. Crislales presentes.

\section{2.- Determinación corrimálica cualitativa.}

De los dalos indicados cn la Tabla l, bajo las condiciones cnsayadas, se puede observar que lodas las cepas miccliales prescntan la capacidad de producir un gran número de curimas o grupos de crizinlis. En toclas las cepas se deicclaron las cur.mas colulolíticas. cilocromo oxidasa. cslerasa. Cosfalasa. lacalsa. y peroxidasa. Por cl contrario, los mayores números de cepas ncgallivas se presentaron en las chrimas tirosimasa (6 cepas). peclinasa (t copas), desoxiribunucleasa, lipasa. urcasa ( 3 ccpas), amilolílicas, oxidasa cxtracelulary protcolílicas ( 1 ccpa). 


\section{DISCUSION}

Los Agaricales, a diferencia de otros grupos de hongos, han sido cscalsamentc esludiados cu su cslado micelial, ya sea desde el punto de visla morrológico. así como bioquímico. El estudio de su fase micclial constituye un nucro enfoque cscasamente conociclo a pesar de que permite un trabajo más permanente. sin la necesidad de esperar que el hongo fructifique.

El aislamiento de cepas miccliales en cultivo puro de Agaricalcs. pucde realizarse a partir de los sustratos colonizados. de estructuras vegelativas y propagativas, o bien a partir de basidiocarpos jórenes y cn bucn estado, disminuyendo con eslo posibles conlaminantes. Este úllimo método presenta la rentaja de conocer cicrlas estrucluras de la especie en aislamiento. De acuerdo a nucstros resullados. algunos de los caracicres prescnles en los basidiocarpos lambićn se desarrollaron en cultiro. Así por cjemplo. aquellos cuyas hifas presculaban ribulas, los cullivos obtenidos de cllos las desimrollaron. Pantidon et al. (1983) inclican la imporlancia laxonómica de este carácler a nivel de especie y' que con nuestro caso fue ulilizado para determinar la purcza de los aislamicntos. En al gunos Agaricales, las hifas de los basidiocarpos no presentinn fibulas como ocurre con Pluteus numus. carácler que lampoco se desarrolló en la cepa micclial. A su ve\%. la cepa aislada de Anthracophyllum discolor desarrolló hifas pigmentadas. similares a las que se encuentran constiluyendo la trama del basidiocarpo (Singer. 1986).

Por el contrario, los cultivos de algunas de las cepas miceliales desirrrollaron estructuras no obscrvaldas en los basidiocarpos. Las hifas de la cepa obicnida de Collybia butyraceu prescnlaron fibulación múltiple, cslc cirrícter ha siclo descrito en cultivo de ciertas especies de Aphyllophorales por Stalpers (1978). Los cultivos de Collybia grinbergsii y Pleuroflamula croceosanguinea desarrollaron hifias esqueléliciss. De acuerdo a Cucras \& Herrera (1971). la formación de hifas csqueléticas sería inducicla por la concentración cle ciertos nutrientes presentes en el medio de cultivo. tales como la dextrosil.

En relación al desirrollo de los micelios en cul tivo puro, Ios medios PDA y AEM permiticron un buen crecimicnlo de las cepas miccliales de $A g r o c i b e$ praecox: C. butyracea. Descolea antarcticay $P$. crocensanguinea. micntras que Pancellus longinquas y P. namus prescntitron un escaso crecimicnto. Sobre C\%Á lodos las cepas miccliales mostraron un escaso creciniento. Algunos de los faclores que podrían limitar el desarrollo de los micelios de Agaricales ell cullivo, hain sido comentides con anterioridad por Pelíce et al. (1992). Garnica (1995), Garnical el al. (1997) y Valcuzrucla et al. (1997).

Con respecto a ha presencia de estructuras de reproducción y resistencia. clestaca el desirrollo de artroconiclios en los micelios de las cepas de: $A$. praecox, D. antarctica y $P$. croceosanguinea, y de clamidosporas en $C$. grinbergsii. Según Watling (1979) y Kendrick \& Watling (1979), este tipo de estructuras son frecuentes en cultivos de Agaricales. A su vez, la cepa micelial de P.nanus desarrolló rizomorfos en cultivo, además presentó caracteres macro-microscópicos semejantes a los descritos para cepas miceliales pertenecientes al género Armillariella (Nobles, 1948; Garnica, 1995; Garnica et al. 1997).

En cuanlo a la caracterización enzimática cualitativa. la Tabla 1 muestra la capacidad que presentan algunos de los micelios cle producir enzimas sobre medio sólido. Los resultados indican la presencia de aquellas enzimas implicadas en la degradación de la lignina como son la lacasa y peroxidasa, en lodas las cepas miceliales de los longos esludiados y sólo la cepa de $A$. praecox resulló negativa para oxidasa cxtracclular. Al respecto, Silva et al. $(1990)$, indican que algunos hongos producen canlidades de polifcnoloxidasas no detectables por este test. Dentro de las cepas que produjeron enzimas lignolíticas se encuentra la obtenida a partir de basidiocarpos de $D$. antarctica, esle agarical ha sido lambién descrito como eclomicorrizico en varias especies de Nothofagus (Garrido. 1988; Singer, 1986).

Por olra parte, se observó la diferente capacidad que tienen las cepas miceliales en utilizar las fuentes de carbono y' nitrógcno emplcadas en el ensayo. La producción de enzimas capaces de clegradar compuestos nilrogenados como son ácidos nuclcícos, protcínas y urea, son imporlantes en los hongos de la madera, ya que los sustratos lignocclulósicos generalmentıe presentan bajos contenidos ell nitrógeno (Micalcs, 1992).

El aislamiento de cepas micelialcs de varias especies de Agaricales, y su posterior caracicrización podría permitir su uso no sólo con fines laxonómicos, sino también como importanles fucnics de metabolitos.

Con respeclo a la presencia de estrucluras de reproducción y resistencia, deslaca el desarrollo de artrosporas en los micelios de las cepas de: $A$. praecox, D. antarctica y $P$. croceosanguinea, y de clamidosporas en $C$. grimbergsii. Scgún Walling (1979) y Kendrick \& Walling (1979) este lipo de estrucluras son frecuentes en cullivos de Agaricales. A su vez, la cepa micelial de P.nanus desilrrolló rizomorfos en cultivo, además presentó caracteres macro-microscópicos semcjantes a los descrilos para cepas micclialles pertenecientes al géncro Armillariella (Nobles, 1948; Garnica. 1995: Garnical el al. 1997).

En cuanto a la caracterización enzimática cualitativa, la Tabla I muestra la capaciclad que presentan algunos de los micelios de producir enzimas sobre medio sólido. Los resultados indican la presencia de aquellas enzimas 
TABLA 1

Detección qualitativa de enzimas en cepas miceliales de Agaricales sobre medios de cultivos sólidos.

\begin{tabular}{|c|c|c|c|c|c|c|c|c|c|c|}
\hline Enzimas/Cepas & 480 & 474 & 442 & 493 & 405 & 265 & 280 & 240 & Tv & Fo \\
\hline Amilolíticas & + & + & + & - & + & d & + & + & - & + \\
\hline Celulolíticas & + & + & + & + & + & + & + & + & + & + \\
\hline Citocromo oxidasa & + & + & + & + & + & + & + & + & + & + \\
\hline Desoribonucleasa & + & + & - & - & + & - & + & $\mathrm{d}$ & - & + \\
\hline Esterasa & + & + & + & + & + & + & + & + & + & - \\
\hline Fosfatasa & $\mathrm{d}$ & + & + & + & + & + & + & + & + & - \\
\hline Lacasa (p-cresol) & + & + & + & + & + & + & + & + & + & - \\
\hline Lacasa(benzidina) & + & + & + & + & + & + & + & + & + & - \\
\hline Lipasa & + & - & + & + & - & + & - & + & + & - \\
\hline Oxidasa ext. & - & + & + & + & + & + & + & + & + & - \\
\hline Pectinasa & - & - & + & - & + & - & + & + & - & + \\
\hline Peroxidasa & + & + & + & + & + & + & + & + & + & - \\
\hline Proteolíticas & + & + & + & - & + & + & + & - & - & + \\
\hline Tirosinasa(p-cresol) & - & - & - & d & $d$ & - & - & - & - & - \\
\hline Tirosinasa(tirosina) & - & - & - & d & $d$ & - & - & - & - & - \\
\hline Ureasa & + & - & - & - & $d$ & + & + & + & - & + \\
\hline
\end{tabular}
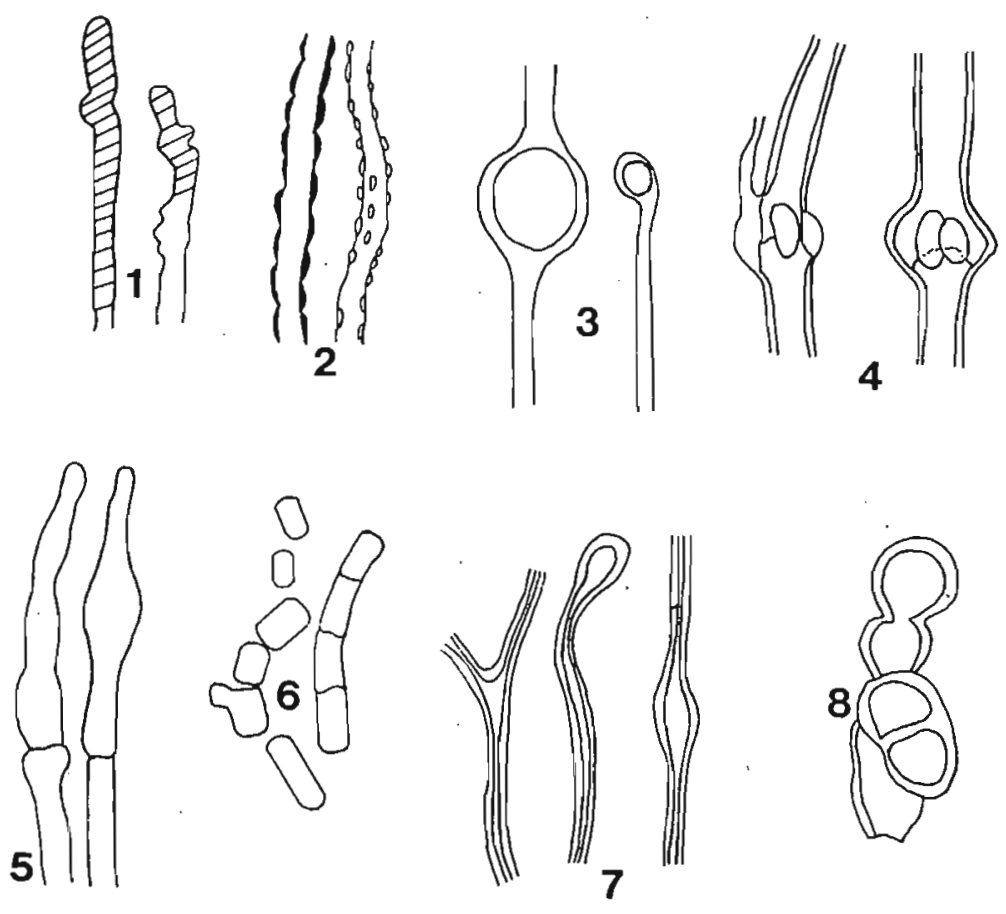

Figuras. 1.- Hifas pigmentadas, 2.- Hifas incrustadas, 3.- Clamidosporas, 4.- Fibulación múltiple, 5.- Gloeocistidios, 6.- Artroconidios, 7.- Hifas esqueléticas, 8.- Hifa monilioide. Escala barra $=10 \mu \mathrm{m}$ 
implicadas en la degradación de la lignina como son la lacasa y peroxidasa, en lodas las cepas miceliales de los hongos estudiados y sólo la cepa obrenida de $A$. praecox resultó negativa para oxidasa cxtracclular. Al respecto. Silva et al. (1990) indican que algunos hongos producen cantidades de polifenoloxidasas no detcclables por este test. Dentro de las ccpas que produjcron cnzimis lignoliticas se encuentra la obienida a partir de basidiocarpos de $D$. antarctica. este agarical ha sido lambićn descrito como ectomicorrízico con varias espccics de Nothofagus (Garrido. 1988: Singer. 1986).

Por olra parte. se observó la diferente capacidad que tienen las cepas micelialcs en utilizar las fuentes de carbono y nitrógeno cmpladas en el ensayo. La producción de enzimas capaces de degradar compuestos nilrogenalos como son ácidos nucleícos, protcínas y urea, son importantes en los hongos de madera, ya que los sustratos lignocelulósicos generalmente presentan bajos contenidos en nitrógeno (Micales, 1992).

El aislamiento de cepas miceliales de varias especies de Agaricales, y su porterior caracterización podría permitir su uso no sólo con fincs taxonómicos, sino que además, los micelios de muchos de estos hongos podrían ser im

\section{AGRADECIMIENTOS}

Queremos agradecer a los proyectos DID S-98-28 Universidad Austral de Chile y FONDECYT 1970707 por el financianiento para el estudio de los Agaricales de la zona valdiviana. S. Garnica desca agradecer al Deutschen Akademischen Austauschdienst (DAAD), la beca concedida para la realización del doctorado en la Eberhard-KarlsUnvicrsiläl Tübingen (Alcmania).

\section{REFERENCIAS}

C'ucvas, F..J. \& Herrera, T. (1971), Variaciones morlologicals de los micelios de Psilocibe maliercola y P'siloci)be zaponecorum en diversos medios de cullivo. Bol. Soc. Mex. Mic. 5:37-46

Garnica, S. (1995). Caracterizacion morlological y biogumica de micelios oblenidos en cultivo puro de bassidiocarpos de Agaricales sensu lato lignoceluloliticos. Tesis de Magister, Esculela de Graduildos, Facultad de Ciencias, Universidad Ausiral de Chile, Valdivia.

Garnica,S.; Ramíres,C. \& Valenzucla,E. (1997). Estudio macromicroseopico y enzimático cualitativo de cepas miceliales de basidiocarpos de Agaricales I. Bol. Nicol. 12:63-73

Garrido,N. (1988). Agaricales s.l, und thre mykorthizen in den Norhofagus-Waldern Miltelchiles. J. Cramer. Berlin-Situltgart.

IIankin, I.. \& Anagnostakis,S.L. (I975). The use ol solid media for detciton of enzime production by fungi. Myeologia 67: $597-$ 607

Kendrick,B. \& Watling,R. (1979). Milosporas in Basidiompcetes. In: "The Whole Fungusm. Kendrick. B. (Edit.) National Muscum of Cantada for the Kananastis Fundation Vol 11 pp.453-473

Mac Faddin,J.F. (1976). Bioclamicial tests lor identilication of medical bacteria. The Wrilliams \& Wilkins Company, Ballimore.

Micales, J.At (1992). Prolcinalses of the brown-rot lungus Postia placenta. Mycologial 84:815-822

Molina,R. \& Palmer,J.G. (1982). Isolation, maintenanee and pure manipulation of ectomycorrhizal lungi. In: "Methods and principles of mycorthizal researclon. Selenck. N. C. (Edit.) The American Plyytopathological Socjely; Floriclal pp. 115-129

Nobles,k.N. (1948). Studies in lorests pathology VI. Identiliciation of cultures of wood-rotling fungi. Call. J. Res., Nect. C. 26:281-431

(1958). Cultural characters as a guide to the taxonomy and phylogeny of the Polyporacean. Cinn. 1. Bot. 36:883-926

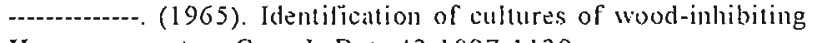
Hymenomyetes. Call. J. Bot. 43:1097-1139

Pantidou,M.; Watling,R. \&Gonou,R.(1983). Mycelial characters, anamorplss, and teleomorplis in genera and species of various families of Agaricales in culture. Mycolaxon Vol XVII: 409-432

Peline,F, Diez,N.T. \& Calonge,F.D. (1992). Obtención de cultivos puros de Basidiomycotina a partir de carpóloros recogidos en Espanīia. Bol. Soc. Micol. Madrid. 17:153-165

Pochon,J. \& Tardicux,p. (1965). Técnicas de anilisis en microbiologial del suelo. Editorial T. E. I. (Técnica e Investigación), Burgos.

Silva,II., Landa,A. \& Agosin,E. (1990). Aislamiento, selección y caracterización de hongos lignoliticos chilenos. Arch. Biol. Med. Exp. 23:41-43

Singer,R. (1986). The Agaricales in modern laxonomy. Koellz Scientific Books, Chicago.

Stialpers,J.A.(1978). Identilication of wood-inhibiting Aphyllophorales in pure culcure. Centratbureatu voor Schimmel Cultures Balarn. Stud. in Mycol. 16:1-248

Thylor,T.13. (1974). Biochemical tests for identilication of mycelial cullures of Basialiomycetes. Ann. Appl. Biol. 78:113-123

Valenzucha,E., Ramirez,C. \& Garnici,S. (1997). Macromicroscopic and fualitalive enzymatic characterization of mycelial strains obtained from balsidiocarps of Mycent species (Agaricales) in Chile. Rev. Hist. Nalt. 70:521-530

Watling, R. (1979). The morphology: variation and ecological signilieance of anamorphs in the Agaricales. In: "The Whole Fungus». Kendrick, B. (Edit.) National Museum of Canada for the Kanthatakis Fundation Il pp.453-473 